\title{
Pobreza y tecnologías de la informacion
}

\author{
Carmen Agustín Lacruz \\ Departamento de Ciencias de la Documentación \\ e Historia de la Ciencia \\ Universidad de Zaragoza \\ Margarita Torrejón Lasheras \\ Centro de Documentación Cáritas. Zaragoza
}

\subsection{Resumen}

Descripción de los diferentes fenómenos de la revolución informacional desencadenada por la aplicación de las tecnologías de la información. Se estudia el valor de la información en relación con los procesos de desarrollo social; y la aparición de redes de comunicación solidaria como elementos útiles para la difusión libre de la información generada y protagonizada por ellas.

Descriptores:: Internet. Revolución Informacional. Pobreza. APC. IPANEX. SPIE.

\subsection{Abstract}

Description of the different phenomena from the informational revolution triggered by the application of the technologies of the information. This article studies the value of the information in relation to the processes of social development, and also the appearance of shared in common communication networks like useful elements for the free diffusion of the information generated and carried out by them.

Keywords: Internet. Informational revolution. Poverty. APC. IPANEX. SPIE.

\section{Introduccion}

En un contexto que se está centrando desde diferentes aproximaciones disciplinares y empíricas en el desarrollo y las características de los sistema de información electrónica y los nuevos documentos, cabe preguntarse qué sentido tiene ocuparse de cuestiones relacionadas con la pobreza y las tecnologías de la información. Las respuestas posibles son variadas y complejas: 
- Quizá todavía se ha hablado y escrito muy poco acerca de la manera en que las nuevas formas de organizar y difundir la información (las nuevas tecnologías) afectan e influyen en problemas como la pobreza, la exclusión social o la marginación.

- Quizá estemos asistiendo a la configuración de una nueva sociedad global de la información en la que vamos a poder contar con unas herramientas potencialmente capaces de crear una sociedad más justa y equilibrada.

- Quizá debamos encontrar entre todos formas de que estas nuevas posibilidades que las tecnologías nos ofrecen no contribuyan a profundizar las diferencias entre las personas y los pueblos, añadiendo nuevos grados de desigualdad y exclusión a los ya existentes y acentuando las distancias entre los inforricos y los infopobres.

- Quizá, finalmente, sea nuestro trabajo como documentalista en el Centro de Documentación de una ONG como Cáritas Diocesana de Zaragoza (en un caso), y como voluntaria en proyectos de desarrollo comunitario y a la par profesional de la documentación (en otro), lo que nos ha llevado a cuestionarnos la forma en que afecta el impacto de las nuevas tecnologías de la información al desarrollo social.

\section{Informacion y pobreza}

De todos es sabido que pobreza no significa únicamente la carencia de bienes materiales (dinero, vivienda, alimentos...) sino también la imposibilidad de acceder a derechos para todos reconocidos, como la salud, la educación, la cultura... Esta imposibilidad viene generada, la mayoría de las veces, por una falta de información sobre dichos derechos; de manera que el desconocimiento de los mismos incapacita a los individuos para su reivindicación y disfrute.

La situación de privilegio que disfrutamos los habitantes del denominado primer mundo, no es lo usual para la mayoría de los habitantes del planeta. Estar en "la onda de Internet" aparece como un lujo propio de los seres más privilegiados. Las estadísticas afirman que el usuario medio de la red tiene entre 30 y 40 años, es blanco, muy cualificado profesionalmente, de cultura occidental y varón.

Por otro lado, en buena parte la comunicación, como el acceso a otros muchos bienes, es un lujo prohibido a los pobres, baste recordar unicamente que un medio de comunicación como el teléfono no ha sido usado nunca por las $2 / 3$ partes de la población mundial, que al menos el $80 \%$ de la población mundial carece de los servicios básicos de comunicaciones y que el $97 \%$ de la investigación científica realizada en el planeta se lleva a cabo en los paises desarrollados, y más de la mitad de ella está relacionada con el armamento. 
En la actualidad la información se genera de forma vertiginosa en soportes muy diversos: nunca la producción editorial ha sido tan elevada, los medios de comunicación (radio, TV...) llegan a un gran número de personas; el número de periódicos crece continuamente. Nunca, como ahora, la información se había convertido en un fenómeno de alcance mundial, y a todo ello, se suma la explosión de las redes informáticas que hacen posible la conexión de millones de personas a través simples ordenadores personales.

El Informe a la UNESCO de la Comisión Internacional sobre la educación para el siglo XXI, presidida por Jacques Delors (1) señala que la interdependencia planetaria y la mundialización son fenómenos capitales de nuestra época, que ya están actuando y que marcarán su impronta en el siglo XXI. El principal peligro que tal situación plantea consiste en que se ahonde un abismo entre una minoría capaz de moverse en ese mundo nuevo en formación y una mayoría que se sienta sacudida por los acontecimientos e impotente para influir en su destino colectivo, con el riesgo de un retroceso colectivo y de múltiples conflictos.

El advenimiento de esta nueva sociedad de la información comporta profundas consecuencias en múltiples sectores de la vida pública, en la educación, en las formas de trabajo, en las relaciones sociales, en la economía, etc. Uno de los graves riesgos que esta nueva sociedad de la información plantea reside en la creación de una sociedad de dos velocidades, compuesta por los que tienen y los que no tienen nada, en la cual sólo una parte de la población tenga acceso a la nueva tecnología, la maneje con soltura y goce plenamente de sus beneficios (2)

Es evidente que todos aquellos pueblos o grupos sociales que no sepan o no puedan adaptarse al uso de estas nuevas tecnologías quedarán fuera del sistema, generandose así nuevas rupturas y desequilibrios. En algunos casos este "quedar fuera" será debido a la falta de recursos financieros y en otros muchos a una falta de voluntad política. No es dificil prever que los desequilibrios aumentaran entre las sociedades que sean capaces de generar información y aquellas otras que se limiten a recibir la que les viene impuesta desde otros entornos. Dentro de los grupos sociales surgirán tensiones y desigualdades entre los que dominen los nuevos instrumentos y quienes no tengan esta posibilidad, constituyendose entonces sociedades con diversos niveles de desarrollo (3)

En este sentido, Huitema, presidente de la Internet Architecture Board, -organismo encargado de supervisar la evolución tecnológica de la Red-, en su obra Et Dieu crea Internett (4), no cree que la información y su acceso agranden la sima entre los que la posen y los que carecen de ella, sino que para él, la Red se convertirá en un factor de igualdad. La separación entre ambos es ya un hecho en nuestra sociedad, lo que ocurrirá es que será más abundante la información y por tanto más fácil el acceso para un mayor número de personas. La visión de 
Huitema está cargada de buenas intenciones, pero creemos que sesga el enfoque desde la misma raiz.

Aunque la tecnología no determina los procesos sociales, sí afecta profundamente a las condiciones materiales de la sociedad, y por tanto, a sus estructuras. Hemos entrado en un nuevo paradigma tecnológico que diversos autores denominan informacional y que representa una divisoria histórica tan importante como lo fué en su momento la Revolución Industrial.

El prestigioso sociólogo e investigador M. Castells (5) señala que la revolución tecnológica actual está centrada en las tecnologías de la información (microelectrónica, informática, telecomunicaciones, ingeniería genética) y su constitución como sistema difundido y aplicado data de los años setenta. Su primera difusión tuvo lugar en la tecnología militar y en las finanzas internacionales, de ahí pasó a las fábricas a principios de los ochenta, se extendió por las oficinas desde finales de los ochenta, y esta llegando en estos momentos a las salas de estar de nuestros hogares, a través de las llamadas "autopistas de la información".

El desarrollo de estas autopistas informativas plantea dos problemas fundamentales para la sociedad a corto plazo: por un lado las grandes empresas de comunicación, cada una con sus apoyos financieros y políticos están tomando posiciones para la construcción y control de la "autopista”. Lo que está ocurriendo en el mundo de los negocios de las comunicaciones en este momento es el equivalente histórico de la formación de las grandes empresas del automóvil y del petróleo en la primera mitad del siglo XX. Quién gane, cómo y para qué en cada país, condicionará durante décadas nuestra economía, nuestra sociedad y nuestra cultura.

El segundo problema es que el acceso a la "autopista" está esencialmente condicionado por la educación y la información, bienes muy desigualmente distribuidos entre los grupos sociales, las personas y los países, como ya hemos visto con anterioridad. Cuanto más se sepan utilizar estas nuevas herramientas tecnológicas más provecho se obtendrá de ellas, y viceversa. Y este viceversa es el mecanismo de exclusión social de nuestra sociedad inmediata. Por ello la autopista desinformada es el camino a la segregación global y a la apropiación por una élite de la mayor revolución tecnológica de la historia.

Esta sociedad de la información en la que ya estamos instalados, organiza la comunicación y por tanto la cultura, en un universo electrónico audiovisual cada vez más diversificado y adaptado a diferentes públicos, y por tanto más influyente. Pero al mismo tiempo que diversifica las imágenes, concentra el poder de producirlas y la capacidad de emitirlas, haciendo de su virtualidad nuestra realidad.

La sociedad de la información en la que estamos, es también aquella en la que el poder de nuestras tecnologías electrónicas y genéticas amplifica extraordina- 
riamente el poder de la mente humana y hace realidad nuestros proyectos, fantasías, sueños, perversiones y pesadillas. Por ello es a la vez la sociedad de las proezas tecnológicas y médicas y la sociedad de la marginación de amplios sectores de la población, irrelevantes para el nuevo sistema. Por ello, estimula la creatividad de los niños al tiempo que satura su imaginario con videojuegos sádicos. Por que es un retrato de nosotros mismos, en toda su crudeza. Por ello, no podemos desarrollar su dimensión creativa y escapar a sus efectos potencialmente devastadores. Por ello, nos debe hacer replantearnos como debemos reequilibrar nuestro superdesarrollo tecnológico y nuestro subdesarrollo social. (6)

\section{Informacion y ONG's}

Por el conjunto de razones que acabamos de exponer la mayor parte de las ONG's se plantean el acercamiento de las nuevas tecnologías de la información a los colectivos más acallados y desfavorecidos creando instrumentos que sirvan para conseguir este objetivo.

Desde nuestro trabajo en el ámbito del desarrollo social, hemos constatado la necesidad, y por tanto la responsabilidad de procurar que estos colectivos no queden una vez más fuera del reparto de la tarta; en este caso la tarta es la información y el reparto está relacionado con hacer su acceso fácil, democrático y justo. Nuestra actitud al respecto es esperanzada, pues pensamos que las tecnologías de la información planteadas en condiciones de igualdad de acceso para todos pueden convertirse en la llave para hacer posible un futuro planetario más rico, solidario, democrático y sostenible.

Los pasados días 3 al 5 de junio de 1996 la World Information Tecnologie and Services Alliance (WITSA) celebró en Bilbao el X Congreso Mundial de las Tecnologías de la Información. Fué en ese Foro donde la Federación IPANEX, de la que hablaremos más adelante y que agrupa a las redes solidarias españolas, dirigió a los congresistas, en nombre de APC, un saludo en el que decía: "Clamamos ante vosotros: no ensanchéis la brecha entre los inforricos y los infopobres" IPANEX hacía este llamamiento para que fuesen tenidas en cuenta las realidades del planeta en que vivimos: desigualdad de recursos, de calidad de vida, de oportunidades y de esperanzas.

Sí como calcula la empresa IBM, en el año 2.000, 1.000 millones de personas conectadas a INTERNET, no es de recibo que los que ya somos privilegiados en renta y en nivel de vida nos convirtamos en inforricos, en detrimento de los 4.000 millones de personas restantes del planeta que viven en situación de pobreza y a las que relegamos a la condición de infopobres.

Muchas ONG's han reconocido la naturaleza básicamente democrática e igualitaria de las tecnologías de la información que hacen posible la utilización 
de sistemas como el correo y las conferencias electrónicas a través de Internet. La red de redes es utilizada por muchas ONG's para comunicarse entre sí y para enviar y recibir información desde los lugares más remotos del planeta.

En la Cumbre de la Tierra celebrada en Río de Janeiro en 1992 se constató que el modelo de desarrollo actual esta en crisis, y por tanto, en evolución, y reconoció la importante función desempeñada por la información generada en todos los niveles, y especialmente constató la importancia de la información generada "desde abajo hacia arriba", pues permite tomar decisiones o conocer mejor la problemática de los propios implicados, haciendo de ellos sujetos de su propio proceso de desarrollo. Pero esa información generada en la base debe ser difundida y dada a conocer. En este contexto nacieron las Redes Solidarias y fue precisamente en la Cumbre de la Tierra donde por primera vez se utilizaron las conferencias (News) y el correo electrónico (E-Mail) para el intercambio de información y la coordinación de esfuerzos solidarios.

Para conseguir y potenciar un desarrollo sustentable es necesaria la acción colectiva y la comunicación, y para ello es imprescindible adoptar medidas a nivel local, regional, nacional y mundial. Las nuevas tecnologías nos permiten, a través de Internet sobre todo, poner en comunicación pequeñas comunidades con el mundo entero, partiendo de un planteamiento solidario: desde el primer mundo podemos aportar nuestros conocimientos y convertirnos en altavoces (que no portavoces, o suplantavoces) de los que no tienen voz en el concierto mundial. Esto supondría conseguir una auténtica democratización de la comunicación y de la información.

En la Cumbre de la Tierra, en 1992, se creó NGONET para promover el intercambio de información de ONG's en todo el mundo y el proceso de creación y gestión de redes, con especial interés en las necesidades informativas de grupos del Sur, organizaciones de pueblos indígenas, de mujeres y de base. El principal objetivo de NGONET ha sido desde su comienzo aumentar la comprensión y participación de las ONG's en cuestiones de medioambiente y desarrollo. Esta organizada en nodos regionales con sede en Montevideo, Nairobi y Penang, vinculados entre sí y conectados con las principales redes internacionales a través de herramientas de comunicación electrónica.

\section{Asociacion para el progreso de las comunicaciones}

Con anterioridad a la celebración de la Cumbre de la Tierra y al nacimiento de la NGONET, existía una red global de comunicación e información electrónica al servicio de las ONG's y activistas ciudadanos denominada Asociación para el Progreso de las Comunicaciones (APC). 
La APC surgió en el verano de 1990 agrupando a siete redes que deseaban coordinar el funcionamiento y el desarrollo de esta emergente red global (7). Es la red de computadoras más extensa del mundo, dedicada especialmente a servir a ONG's y a cuantas personas trabajan por el cambio social. Las redes que integran APC comparten una misión común: desarrollar y mantener un sistema informático que permita a grupos geográficamente dispersos coordinar actividades y proyectos para generar el cambio social dentro de una filosofía solidaria. Los costes de utilización de las nuevas tecnologías de comunicación se ofrecen a un precio mucho más bajo que los de tipo comercial.

La APC es una red mundial de miembros asociados de carácter alternativo que se define así misma como herramienta de intercambio de información a nivel mundial para la sustentabilidad ambiental, los derechos humanos y la justicia económica y social. APC es también una ONG con el status de Consultora de la ONU, categoría 1, lo que significa un asiento propio permanente en la Asamblea de Naciones Unidas y el trabajo regular con varias de sus agencias y organizaciones para lograr que los documentos que generan estén al alcance de las ONG's.

En su Declaración de Principios, apartado 3, APC proclama que su objetivo es proporcionar un instrumento de comunicaciones poderoso y efectivo que posibilite la creación de una verdadera sociedad global, el intercambio de información y la toma de decisiones a nivel internacional. APC busca proveer redes de comunicación electrónicas globalmente interconectadas, comprometidas con un flujo de información libre y equilibrado. Las organizaciones miembros de APC, sirven a quienes trabajan a favor de metas como la paz, la prevención de la guerra, la eliminación del militarismo, la protección del medio ambiente, la erradicación de la pobreza, la promoción del desarrollo sostenible y equilibrado, la salud, la educación, el avance de la democracia participativa y la resolución no violenta de conflictos.

Las redes APC constituyen el más grande foro internacional utilizado por ONG's, personas físicas o jurídicas, individuos o grupos, y diferentes organizaciones sociales sin ánimo de lucro que trabajan por el cambio social. Estas redes APC se distinguen por la profundidad y calidad de sus recursos de información, por el alcance global de sus servicios, y por la diversidad de sus miembros. Hoy, APC está constituida por 22 redes integrantes y cerca de 50 redes asociadas, que sirven a más de 40.000 usuarios de 133 países. APC proporciona a muchas de estas redes asociadas el único acceso por correo electrónico de las ONG's con sus países respectivos.

APC ha estado presente en todas las Cumbres que se han celebrado desde 1992 a nivel mundial: 
- Conferencia de Derechos Humanos en Viena en 1993,

- Conferencia de Población y Desarrollo en el Cairo en 1994

- Cumbre Mundial de Desarrollo Social en Copenhagen,

- Conferencia de las Partes de la Convención sobre el Clima en Berlín y

- Cuarta Conferencia Mundial de Mujeres en Beijing.

En estos momentos las Redes APC ofrecen a sus usuarios las herramientas telemáticas habituales: correo electrónico, videoconferencias privadas y públicas, acceso a diferentes bases de datos, herramientas de Navegación en Internet, servicios de información y noticias y directorio internacional de usuarios.

\section{IPANEX: Federacion española de redes solidarias (URL=http://www. ipanex.apc.org.)}

En España las redes solidarias están agrupadas en una federación llamada IPANEX que agrupa a: PANGEA (Cataluña), NODO 50 (Madrid), EUSNET (Euskadi) y XARXANETA (Cataluña).

\subsection{NODO 50 (URL=http:// www.nodo50.ix.apc.org.)}

Se puso en marcha en Madrid en octubre de 1994 para facilitar el intercambio de información entre grupos locales e internacionales y así coordinar la campaña contra las políticas del Banco Mundial y del Fondo Monetario Internacional. Paralelamente las ONG's celebraron un Foro alternativo y allí empezó a funcionar.

NODO 50 es miembro de pleno derecho de APC y como la mayoría de los Nodos alternativos y solidarios organiza su información de la siguiente manera:

- Organizaciones y grupos que componen el nodo; cada uno de los cuales incluye una página WEB con las características propias de la organización, actividades, programas, filosofía, etc.

- Publicaciones que se pueden consultar;

- Convocatorias a diversos actos, seminarios, jornadas, manifestaciones, etc.

- Enlaces que tienen con APC y en general con toda la RED.

A menudo aparece un apartado dedicado a la formación de los integrantes del nodo: cómo hacer páginas en html o un cursillo básico sobre INTERNET.

Los integrantes de NODO 50 son ONG's y organizaciones sin ánimo de lucro que incluyen información sobre cooperación y desarrollo del Tercer Mundo, medioambiente, economía solidaria, etc.

Desde NODO 50 se puede acceder a todas las conferencias de APC/IPANEX y a los grupos de noticias y de discusión de la propia APC. Cabe destacar el gru 
po de conferencias de Amnistía Internacional y los grupos de noticias sobre Derechos Humanos.

\subsection{PANGEA ( http:// www. pangea.org.)}

Es un nodo alternativo de Barcelona integrado en APC, y puesto en marcha por la organización Cooperación para la Cooperación, la federación de asociaciones civiles sin ánimo de lucro, y cuenta con el apoyo técnico de la Escuela Técnica Superior de Ingeniería de Telecomunicaciones de Barcelona.

Pangea es un servidor trilingüe con estructura y contenido similar al de NODO50,. Está integrado por más de un centenar de organizaciones dedicadas a la intervención social, la solidaridad y la cooperación internacional. Ofrece bases de datos de gran calidad sobre ONG's españolas y un apartado dedicado al ámbito educativo con especial atención a las cuestiones de renovación pedagódica, educación para la paz e interculturalidad

\subsection{EUSNET (htttp:// www.eusnet.org.)}

Es un servidor integrado dentro de IPANEX y de APC, asentado en el País Vasco y Navarra. Es un servidor bilingüe euskera-castellano con estructura y propósitos similares a los que hemos visto en NODO50 y PANGEA. Incluye diferentes colectivos relacionados con la cooperación internacional y el desarrollo solidario.

EUSNET ofrece una gama muy amplia de buscadores y enlaces ordenados temáticamente. Incorpora enlaces a los principales medios de comunicación escrita tanto de España como del extranjero; además ofrece enlaces a emisoras de radio y a varias publicaciones periódicas. Es interesante la información que ofrece en el apartado denominado "Las otras voces del planeta".

\subsection{XARXANETA (http://www.xarxaneta.org.)}

Pertenece a APC y a IPANEX. Es una ONG cuyo ámbito de trabajo es Cataluña, Valencia y Baleares, y que tiene como objetivo es prestar servicios avanzados de telecomunicación a bajo coste a las organizaciones e individuos que trabajan en la lucha contra la marginación social y a favor del desarrollo integral de pueblos y personas.

Incluye enlaces dirigidos fundamentalmente a temas de antimilitarismos, minorías étnicas, SIDA, mujeres y solidaridad internacional. Ofrece también un apartado desde donde se accede a publicaciones con información alternativa.

\section{Una red solidaria aragonesa: SPIE}

El Servidor Popular de Información Electrónica de Aragón es el nombre de 
una organización sin ánimo de lucro integrda por ONG's de ayuda al desarrollo, pro derechos humanos, con sensibilidad mediambiental y social, formada para gestionar un servidor de comunicaciones destinado a difundir informaciónes sobre temas de desarrollo, cooperación, ecología y derechos humanos.

Nació en 1995 fruto del trabajo cooperativo de un grupo de ONG's radicadas en Aragón de ámbito regional y nacional, con intención de asociarse a IPANEX y de constituirse en nodo de APC en Aragón.

SPIE esta integrada por las siguientes ONG's:

- Acción Solidaria Aragonesa (8)

- AEDENAT-ECOFONTANEROS (9)

- Agenda Latinoamericana de Teología (10)

- Cáritas Diocesana de Zaragoza (11)

- Comité de Solidaridad Internacionalista (12)

- Comité de SolidaridadOscar Romero (13)

- Fundación Ecología y Desarrollo (14)

- Ingeniería sin Fronteras (15)

- INTERMON (16)

- Medicus Mundi (17)

- Movimiento Por la Paz el Desarme y la Libertad (18)

- Seminario de Investigaciones para la Paz (19)

- Federación de Asociaciones de Barrios de Zaragoza

Los estatutos de la asociación recogen los principios básicos en los que se inspira el proyecto:

1. Ser un servicio alternativo de información.

2. Facilitar la intercomunicación de los colectivos populares y ONG's.

3. Fomentar la cultura de la solidaridad.

4. Construir puentes de información entre el Norte y el Sur.

5. Fomentar la lucha por los derechos humanos para conseguir un cambio social que signifique la igualdad de todos los seres humanos.

6. Trabajar por la paz, prevención de la guerra, eliminación del militarismo, protección del medio ambiente, promoción del desarrollo sostenible, promoción del derecho a la salud y a la educación, luchar por el avance de la democracia participativa y aumentar la resolución no violenta de conflictos. 
El trabajo del Proyecto SPIE está presidido por un código ético aceptado por todos los miembros de manera que nadie puede difundir información que no respete estos cuatro principios: no violencia, no sexismo, no racismo y no uso comercial.

La estructura del servidor recoge: Agenda de actualidad, Información de las organizaciones participantes, Recursos en Internet, Publicaciones, Información sobre el Proyecto SPIE. Son también muy importante las secciones de novedades, de información, de conflictos vivos en el mundo y la conexión con los nodos de IPANEX y APC en América.

El trabajo y coordinación del Proyecto SPIE está desarrollado por voluntarios. Existe un coordinador general, un coordinador técnico y un coordinador editorial. Las tareas de apoyo técnico son llevadas a cabo por el equipo de Ingeniería Sin Fronteras.

SPIE se ha convertido en la actualidad en un lugar útil, en una plaza mayor virtual en la que diferentes tipos de asociaciones ciudadanas dan a conocer sus trabajos, los intercambian y comentan.

\section{Notas}

(1) Delors, Jacques. La educación encierra un tesoro.

(2) Bangemann

(3) Delors, Jacques. Op.Cit.

(4) Et dieu crea internet. 1995

(5) Castells, M. La autopista desinformada. El Periódico de Cataluña. 7 de enero de 1995; y La sociedad de la información. El País. 25 de febrero de 1995

(6) Castells, M. La sociedad de la información. Temas para el debate, 1995.

(7) Entre 1982 y 1987 surgieron varias redes de comunicación electrónica sin ánimo de lucro al servicio de la información y comunicación. En 1987, GreenNet en Inglaterra comenzó a colaborar con el Instituto para la Comunicación Global (IGC), que ofrece PeaceNet, EcoNet, ConflictNet y LaborNet en Estados Unidos. Estas dos redes comenzaron a compartir conferencias y demostraron que las comunicaciones transnacionales pueden ser de gran utilidad para comunidades, tanto locales como internacionales, que trabajan en pacifismo, derechos humanos y medio ambiente. Fué tal el éxito que a finales de 1989 redes en Suecia (NordNet), Canada (Web), Brasil (AlterNex), Nicaragua (Nicarao) y Australia (Pegasus), comenzaron a intercambiar información entre sí, con IGC y con GreenNet.En el verano de 1990, estas 7 redes fundaron la Association for Progressive Communications (APC).

(8) Accion Solidaria Aragonesa (ASA), es una ONG de cooperación internacional que trabaja por el desarrollo de los pueblos del sur, buscando y analizando las causas de la desigualdad entre el primer y tercer mundo sensibilizando a la población en la búsqueda de respuestas solidarias y activas

Scire. 3 : 2 (jul.-dic. 1997) 131-144 
(9) AEDENAT-Ecofontaneros, es una ONG ecologista que trabaja en la denuncia de situaciones y acciones que atentan contra el medioambiente a nivel regional y estatal. En su página Web incluye enlaces con Greenpeace International.

(10)Agenda lationamericana de Teologia, es una publicación anual de la Universidad Centroamericana de Managua. Su director es José $\mathbf{M}^{\mathrm{a}}$ Vigil, teólogo y profesor de dicho centro, auténtico alma mater del proyecto SPIE.

(11)Caritas Diocesana, ONG perteneciente a la Iglesia Católica que desarrolla su trabajo sobre la Teología de la Caridad, atraves de programas que actuan para erradicar la pobreza, interviniendo en las causas que la general y ayudando a la promoción humana y social de las personas. Desarrolla proyectos de actuación con niños, inmigrantes, transeuntes, presos, mujeres, parados y toxicómanos. También desarrolla programas de cooperación con el tercer mundo.

(12)Comite de solidaridad internacionalista, nace como fruto de la reorganización de uno de los grupos pioneros de solidaridad con centroamérica, el Comité de Solidaridad con Nicaragua. Es una ONG que documenta, denucia y difunde la situaciones de injusticia y explotación de los pueblos y naciones, y promueve actuaciones de apoyo. Trabaja en Guatemala, Cuba, El Salvador y Nicaragua, y lleva a cabo tareas de divulgación y sensibilización

(13)Comite de solidaridad Oscar Romero, es un grupo cristiano que desarrolla trabajos de concienciación solidaria para abrir la visión eurocéntrica a realidades del tercer mundo, especialmente América Latina.

(14)Fundacion Ecologia y Desarrollo, incluye a profesionales aragoneses comprometidos con el desarrollo sostenible de nuestro país y del planeta. Su objetivo es proponer alternativas viables y sostenibles desde una actitud dialogante y firme. Sus miembros opinan que pensar es comprometerse y se consideran en deuda con una sociedad global que les ha dado medios para su formación, a través de su compromiso quieren saldar era deuda.

(15)Ingenieros sin fronteras, es una organización de cooperación técnica para el desarro1lo. Entienden la cooperación como un enriquecimiento mútuo: económico, cultural, medioambiental y humano, y rechazan cualquier enfoque paternalista. Proponen que las comunidades tienen que ser sujetos protagonistas de su propio desarrollo. Desarrollan proyectos técnicos, lineas de investigación, debate y colaboración.

(16)INTERMON, es una Fundación que trabaja para erradicar la pobreza de las poblaciones del Tercer Mundo y proporcionarles los medios para que puedan alcanzar su propio desarrollo. Realiza proyectos en 28 paises de Africa, América Latina y Asia.

(17)Medicus Mundi es una ONG que se dedica a la Cooperación Sanitaria para el desarrollo del tercer mundo y de las poblaciones marginadas, empleando a profesionales sanitarios que colaboran de forma voluntaria. Es la rama española de la ONG mundial del mismo nombre. Medicus Mundi Aragón trabaja en Peru, Bolivia, República Dominicana y Mozambique

(18)Movimiento por la paz El desarme y la libertad, es una ONG de que tabaja por la convivencia pacífica entre los pueblos y para minorar los efectos de la guerra y los desas 
tres naturales en la población civil. Para ellos es prioritario no tomar partido por ninguna de las partes en conflicto.

(19)Seminario de investigaciones para la paz, su objetivo es contribuir de manera interdisciplinar a la investigación de la paz en sus múltiples facetas. Desarrollo sesiones de estudio y debate, promueve investigaciones, emite informes y propuestas para que lleguen a la opinión pública, y convoca anualmente las Jornadas Aragonesas de Educación para la Paz.

\section{Bibliografia.}

Bissio, R. (1994). Cyberespace et démocratie. // Le Monde diplomatique. (julio 1994) 1617

Bissio, R. La cibernética y los discapacitados de la "aldea global". // Guia del Mundo 1996-1997: El mundo visto desde el sur. Madrid : IEPALA, 1996.

Buceta Facorro, Luis (1997). Las nuevas tecnologías al servicio de la "comunicación". // Documentación Social. Julio-septiembre, 108 (1997) 249-256.

Bustamante Donas, Javier (1997). La revolución del conocimiento y la nueva cultura digital. // Documentación Social. (julio-septiembre 1997, nº 108. Pag. 179-202.

Castells, M. (1995). La autopista desinformada.. // El Periódico de Cataluña. (7 de enero de 1995)

Castells, M. (1995). La ciudad informacional: Tecnologías de la Información, reestructuración económica y proceso urbano-regional. Madrid : Alianza, 1995, p.70-72.

Castells, M. (1995). La sociedad de la información. El País. (25 de febrero de 1995) 11.

Castells, M. (1995). La sociedad de la información. // Temas para el debate. 5 (1995),.

Colomer Busquets, Miquel. Comunicación por ordenador y comunicación alternativa. URL=mcolomer@nodo50.gn.apc.es

Echeverria, Javier (1995). Cosmopolitas domésticos. Barcelona : Anagrama, 1995.

Echeverria, Javier (1996). Internet, la calle mayor de Telépolis. Net conexion. 3 (Enero 1996) 42-45.

Echeverria, Javier (1994). Telépolis. Barcelona : Destino, 1994.

Fernandez Calvo, Rafael (1996). De internautas a ciudadanos. // Web. 2 (enero 1996) 25.

Fernandez Calvo, Rafael (1996). Internautas y ciudadanos de la red (recapitulación). // Web. (mayo 1996) 28-29.

Fernandez Fernandez, Fernando. Socio-ética de la comunicación: Información, comunicación y comunión en el desarrollo humano integral e integrado. // Documentación Social. 108 (Julio-septiembre 1997) 271-290.

Guia del mundo 1996-1997: El mundo visto desde el sur. Madrid : IEPALA, 1996.

Huitema, Christian (1995). Et Dieu créa Internet. Paris : Editions Euyrolles, 1995.

Informe sobre desarrollo humano 1996 : Publicado para el Programa de las Naciones Unidas para el Desarrollo (PNUD). Madrid : Mundi-Prensa, 1996.

Irantzu Larrañaga ; et al. (1996). Internet solidaria: la última revolución. Tafalla : Txalaparta, 1996.

Joyanes Aguilar, Luis (1997). La galaxia Internet: la última utopía. : Condicionantes y

Scire. $3: 2$ (jul.-dic. 1997) 131-144 


\section{Carmen Agustín y Margarita Torrejón}

apuestas. // Documentación Social. 108 (julio-septiembre, 1997), 71-102.

La Educacion encierra un tesoro: Informe a la UNESCO de la Comisión Internacional sobre la educación para el siglo XXI, presidida por Jacques Delors (1996). Madrid : Santillana ; UNESCO, 1996.

Lorn Norman, Alfred (1993). Informational society: an econommic theory of discovery, invention and innovation. Londres : Kluwer Academic Publisher, 1993.

Martin Garcia, Victor (1997). La Edad de la Informática. La cibersociedad. // Documentación Social. 108 (Julio-septiembre, 1997) 11-32.

Molins, Mercé (1996). Internet, arma de guerra. // Web. 6 (Mayo 1996) 74-78.

Negroponte, Nicholas (1995). El mundo digital. Barcelona : Ediciones B, 1995.

Rouille D’Orfeuil, Henri (1994). El tercer mundo: claves de lectura. Santander : Sal Terrae, 1994

Terceiro, José B. (1996). Socied@d digit@1. Del homo sapiens al homo digitalis. Madrid : Alianza Editorial, 1996.

Trejo Delarbre, Raúl (1995). La nueva alfombra mágica:usos y mitos de Internet, la red de redes. Madrid : Fundesco, 1995. 